\title{
Temporal Dynamics of Parvalbumin-Expressing Axo-axonic and Basket Cells in the Rat Medial Prefrontal Cortex In Vivo
}

\author{
Lema Massi, ${ }^{1,2 \star}$ Michael Lagler, ${ }^{1,2 \star}$ Katja Hartwich, ${ }^{1,2}$ Zsolt Borhegyi, ${ }^{2}$ Peter Somogyi ${ }^{1,2}$ and Thomas Klausberger ${ }^{1,2}$ \\ ${ }^{1}$ Medical Research Council Anatomical Neuropharmacology Unit, University of Oxford, Oxford OX1 3TH, United Kingdom, and ${ }^{2}$ Center for Brain Research, \\ Department of Cognitive Neurobiology, Medical University of Vienna, 1090 Vienna, Austria
}

Axo-axonic interneurons, innervating exclusively axon initial segments, and parvalbumin-expressing basket interneurons, targeting somata, dendrites, and spines of pyramidal cells, have been proposed to control neuronal activity in prefrontal circuits. We recorded the spike-timing of identified neurons in the prelimbic cortex of anesthetized rats, and show that axo-axonic cells increase their firing during tail pinch-induced brain state-activation. In addition, axo-axonic cells differ from other GABAergic parvalbumin-expressing cells in their spike timing during DOWN- to UP-state transitions of slow oscillations and in their coupling to gamma and spindle oscillations. The distinct firing dynamics and synaptic targets of axo-axonic and other parvalbumin-expressing cells provide differential contributions to the temporal organization of prefrontal networks.

\section{Introduction}

Parvalbumin (PV)-expressing GABAergic interneurons are key regulators of temporal operations in cortical networks. They provide precise temporal control over pyramidal cells and generate synchronous network activity and neuronal oscillations (Tamás et al., 2000; Vida et al., 2006; Cardin et al., 2009; Atallah et al., 2012; Lovett-Barron et al., 2012; Royer et al., 2012). Furthermore, dysfunction in their GABA-mediated synaptic transmission has been reported in patients with schizophrenia and might be a core feature of the disease (Lewis et al., 2005). However, PVexpressing interneurons do not represent a homogenous population, as different types of interneuron express PV; e.g., axo-axonic cells innervate exclusively axon initial segments of pyramidal cells (Somogyi, 1977) and basket cells target somata, dendrites and spines of pyramidal cells (Kubota et al., 2007). Indeed, PV-expressing interneurons with heterogeneous firing patterns have been described (Puig et al., 2008). Do distinct types of PV-expressing interneurons contribute differentially to oscillations and circuit operations in vivo?

Synchronous firing and network oscillations structure the activity of neuronal circuits in the working brain. Slow oscillations with UP- and DOWN-states and nested spindle or gamma oscil-

Received July 20, 2012; revised Sept. 19, 2012; accepted Sept. 27, 2012.

Author contributions: L.M., M.L., K.H., Z.B., P.S., and T.K. designed research; L.M., M.L., K.H., Z.B., P.S., and T.K. performed research; L.M., M.L., K.H., Z.B., P.S., and T.K. analyzed data; L.M., M.L., K.H., Z.B., P.S., and T.K. wrote the paper.

This work was supported by grants SCICO3 of the Vienna Science and Technology Fund, W1205 of the Austrian Science Fund and the Medical Research Council, United Kingdom. We thank Romana Hauer, Kristina Detzner, J. David B. Roberts, and Ben Micklem for excellent technical assistance; and József Somogyi for his exquisite help with fluorescence microscopy signal detection.

*L.M. and M.L. contributed to this work to a comparable extent

The authors declare no competing financial interests.

Correspondence should be addressed to Thomas Klausberger, Center for Brain Research, Department of Cognitive Neurobiology, Medical University of Vienna, Spitalgasse 4, 1090 Vienna, Austria. E-mail: thomas.klausberger@meduniwien.ac.at.

DOI:10.1523/JNEUROSCI.3475-12.2012

Copyright $\odot 2012$ the authors $\quad 0270-6474 / 12 / 3216496-07 \$ 15.00 / 0$ lations are a hallmark of the sleeping brain. Arousal induces smaller amplitude activity including theta oscillations (Buzsáki, 2006). Here, we tested the firing patterns of axo-axonic, basket and other PV-expressing cells during these network operations in the medial prefrontal cortex.

\section{Materials and Methods}

In vivo recording and labeling. Experimental procedures were performed on adult male Sprague Dawley rats (250-390 g) and were performed in accordance with licenses approved by the UK Home Office or Austrian Ministry of Science. Anesthesia was induced with isoflurane in oxygen, followed by urethane $(1.25 \mathrm{~g} / \mathrm{kg}$ body weight, i.p.) for long-term anesthesia, plus additional doses of a mixture of ketamine and xylazine (20 and $2 \mathrm{mg} / \mathrm{kg}$, respectively), as needed. The rat's head was fixed in a stereotaxic frame and all wound margins were treated with the local anesthetic Marcaine. Body temperature was maintained at $37^{\circ} \mathrm{C}$ with a heating pad. Action potentials of single neurons and local field potential (LFP) were recorded extracellularly in layers II-V of the prelimbic area of the prefrontal cortex using a glass electrode (15-25 M 2 ) filled with $1.5 \%$ Neurobiotin in $0.5 \mathrm{M} \mathrm{NaCl}$. Coordinates relative to bregma with a $10^{\circ}$ angle lateromedial were as follows: anteroposterior, $+3.6 \mathrm{~mm}$; mediolateral, $-0.7 \mathrm{~mm}$; dorsoventral, $2.5-3.5 \mathrm{~mm}$. A stationary second glass electrode, also filled with $1.5 \%$ Neurobiotin, was used to record the LFP in stratum pyramidale of the dorsal CAl hippocampus. Coordinates relative to bregma with a $10^{\circ}$ angle caudorostral were as follows: anteroposterior, $-4.1 \mathrm{~mm}$; mediolateral, $-2.2 \mathrm{~mm}$; dorsoventral, $2.2-2.4$ $\mathrm{mm}$ ). The correct placement of the CA1 electrode in the pyramidal layer was confirmed as described previously (Hartwich et al., 2009). In the prelimbic cortex, putative interneurons were distinguished from putative pyramidal cells by the lack of complex spike bursts and narrow spike width (Csicsvari et al., 1998). The signals from the electrodes were amplified and filtered on-line at $0.3-300 \mathrm{~Hz}$ (LFP) and $0.8-5 \mathrm{kHz}$ (action potentials) (BF-48DGX and DPA-2FS, NPI Electronic). Hum-Bugs (Digitimer Ltd.) were used to remove the $50 \mathrm{~Hz}$ noise from the LFP signals. The LFP and units were sampled online at 1 and $20 \mathrm{kHz}$, respectively (Power 1401, Cambridge Electronic Design). Following recording, individual neurons were specifically labeled with Neurobiotin by moving the electrode to a juxtacellular position (Pinault, 1996). All data were acquired and analyzed using the software Spike 2 (Cambridge Electronic 


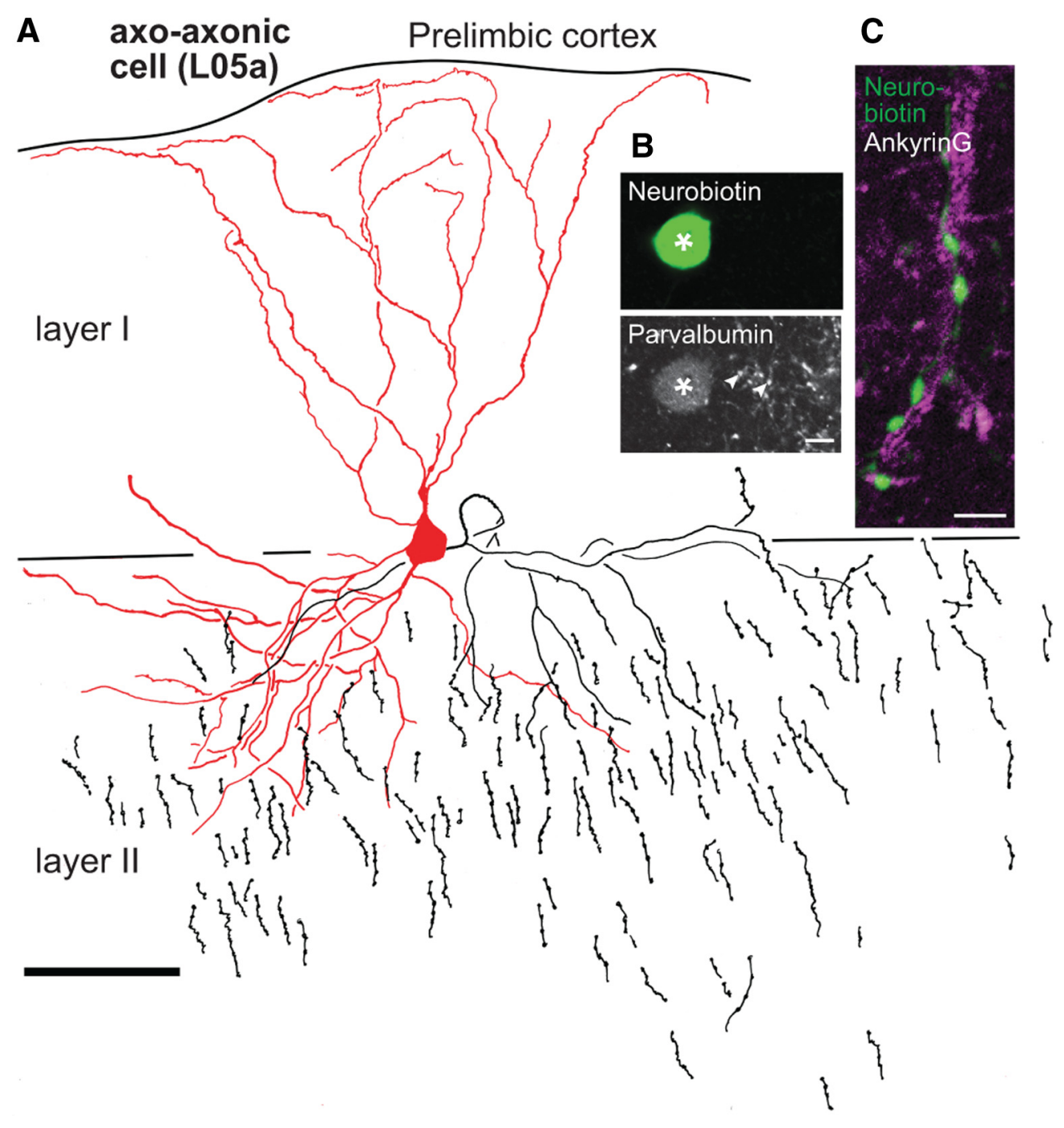

Figure 1. Reconstruction and molecular expression of a recorded and labeled axo-axonic cell. $A$, An axo-axonic cell (L05a) with complete dendritic tree (red) and radial axonal terminal segments (black) from two $70-\mu \mathrm{m}$-thick sections. $B$, Single optical slice confocal microscopic image of the labeled soma (star), which was weakly immunopositive for PV, next to immunopositive boutons (arrows). C, Axo-axonic boutons (green, Z stack) following an axon initial segment labeled for ankyrin-G (purple). Scale bars: $\boldsymbol{A}, 50 \mu \mathrm{m} ; \boldsymbol{B}, 5 \mu \mathrm{m} ; \boldsymbol{C}, 2 \mu \mathrm{m}$.

Design). Methods for tetrode recordings of pyramidal cells were reported previously (Hartwich et al., 2009).

Tissue processing and immunohistochemical analysis. After extracellular recording and juxtacellular labeling, rats were perfused with saline followed by a fixative solution containing $4 \%$ paraformaldehyde, $15 \% \mathrm{v} / \mathrm{v}$ saturated picric acid and $0.05 \%$ glutaraldehyde in $0.1 \mathrm{~m}$ phosphate buffer. The brain was removed and sectioned at $70 \mu \mathrm{m}$ thickness. The labeled cells were visualized by streptavidin-coupled fluorophores and the interneurons were tested by immunofluorescence microscopy with primary antisera to PV raised in guinea pig (1:5000, Synaptic Systems, \#195 $004)$ and ankyrin-G raised in mouse $\left(200 \mu \mathrm{g} / \mathrm{ml} \mathrm{IgG}_{1}, 1: 200\right.$, Santa Cruz Biotechnology, \#sc-12719), which were detected, respectively, by Cy5 (1:250; Jackson Immunoresearch Laboratories) or Dylight405 (1:100; Jackson Immunoresearch Laboratories)-conjugated secondary antibodies. The antiserum to PV detects a band of the expected molecular mass in Western blots, labels cells transfected with parvalbumin-GFP fusion constructs, and labels the same cell populations in the brain as other antibodies to parvalbumin (information: Synaptic Systems). The specificity of the mouse antibodies to ankyrin-G primary antibody has been tested by the manufacturer (Santa Cruz Biotechnology) by Western blot analysis of ankyrin-G expressed in Caco-2 whole-cell lysate. Method specificity was tested by omitting the primary antibodies. Immunoreactions were recorded as image stacks taken using an AxioImager.Z1 microscope ( $63 \times$ or $40 \times$ objectives, LSM 710 scanning head, ZEN 5.0 software) and sequential scanning as described previously (Lasztoczi et al., 2011). Following epifluorescence evaluation, some sections of the labeled cells were visualized by a horseradish peroxidase (HRP) method using avidinbiotinylated HRP and 3,3'-diaminobenzidine as chromogen, followed by 0.5-1\% osmium tetroxide to enhance contrast. After dehydration, sections were embedded in epoxy resin. The somatodendritic and axonal arborizations of a selected axo-axonic cell (L05) were reconstructed using a drawing tube with a $63 \times$ objective. The axonal synaptic targets of two basket cells within layer III were analyzed using electron microscopy, as described previously (Hartwich et al., 2009).

Analysis of the firing patterns. The change in firing activity caused by the tail pinch was determined by comparing the firing rate in a $10 \mathrm{~s}$ window before the pinch with the firing rate during the pinch (Wilcoxon signed rank test).

Slow oscillation periods were detected as data segments with theta $(3-6 \mathrm{~Hz})$ to delta $(2-3 \mathrm{~Hz})$ ratios $<2$ derived from recordings in the stratum oriens or pyramidale of the dorsal CA1 region. Theta-to-delta ratios were smoothed by a sliding average with a length of $5 \mathrm{~s}$ and $50 \%$ overlap. The minimal duration of analyzed data segments was $10 \mathrm{~s}$. Slow oscillations in the medial prefrontal cortex were recorded by the same glass electrode that was used for juxtacellular labeling. The discrimination of DOWN- and UP-states was done by extracting the shape information below $2.5 \mathrm{~Hz}$ after a continuous wavelet transform using a Daubechies (3) wavelet function and by setting the threshold to $\cos (\theta)=$ 
A

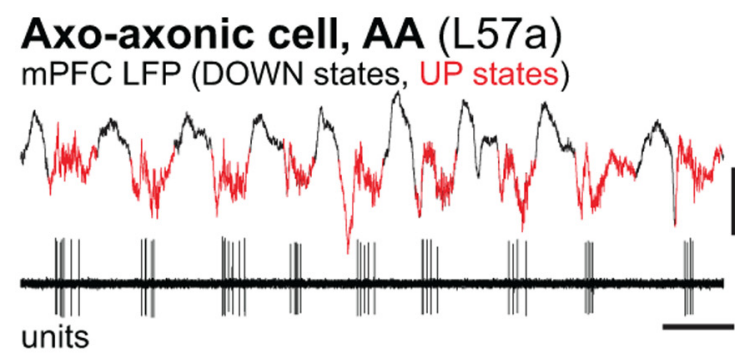

Basket cell, BC (L10c)

mPFC LFP (DOWN states, UP states)

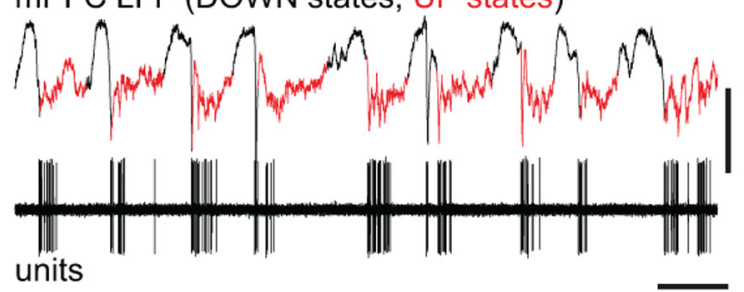

Pyramidal cell, PYR (K161d1.5) mPFC LFP (DOWN states, UP states)

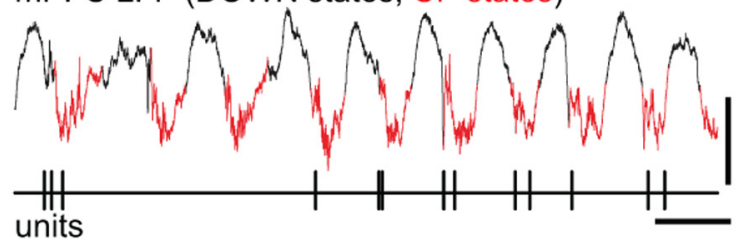

C

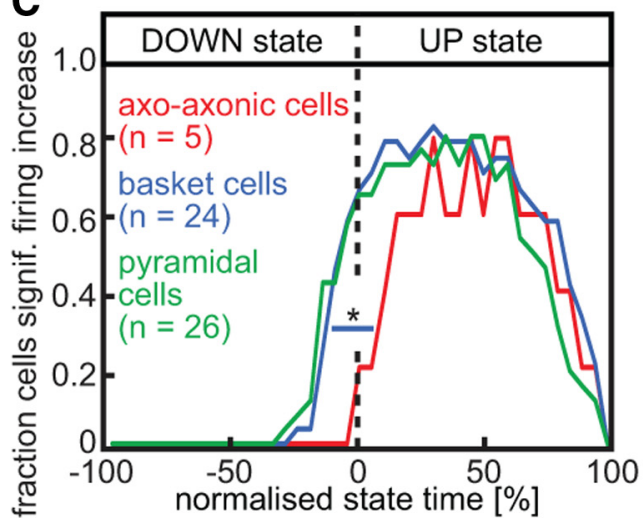

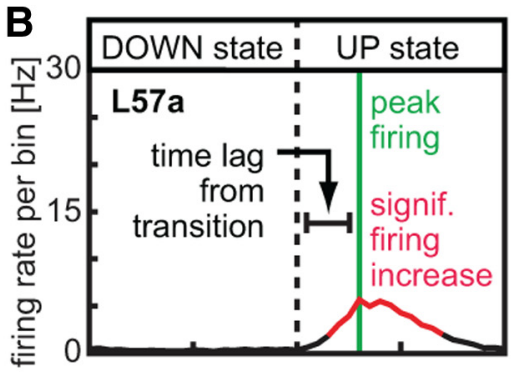
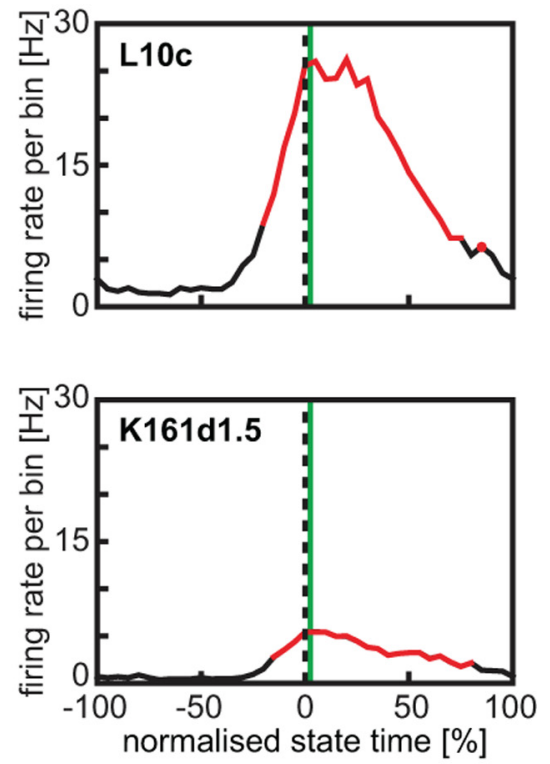

D

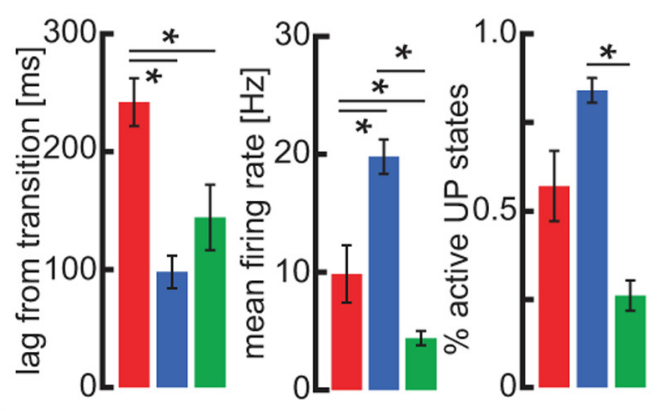

Figure 2. Distinct spike-timing of axo-axonic and basket cells during slow oscillations. $\boldsymbol{A}$, Spike trains of an axo-axonic, basket and pyramidal cell during slow oscillations. $\boldsymbol{B}$, Analysis of peak firing times (green line) and significant firing increase (red) of the cells shown in $A$; the dotted line depicts LFP transitions from DOWN-to UP-states. C, Axo-axonic cells increase their firing significantly later than basket cells during the UP-state ( ${ }^{*} p<0.05$, Kolmogorov-Smirnov test with Holm-Bonferroni correction). $\boldsymbol{D}$, Time lag from LFP-state transition to peak firing; mean firing rates during UP states and proportion of UP-states with firing for the three cell types (color code same as in C) was analyzed with Kruskal-Wallis ANOVA, multiple comparisons with Bonferroni adjustments ( ${ }^{*} p<$ 0.05). All values shown as mean \pm SEM. Calibration: $A, 1 \mathrm{~s}, 1 \mathrm{mV}$.

0 . This wavelet transform was confirmed not to cause a phase shift, and similar results were obtained by using a complexed Morlet (1-1) wavelet function. Periods with $\cos (\theta)<0$ were considered as DOWN-states and periods with $\cos (\theta)>0$ as UP-states. States shorter than 300 ms were rejected. State differentiation was based on the LFP recorded in the medial prefrontal cortex, except for recordings of the cells L05a and L58a, in which DOWN- and UP-states could only be reliably separated in the hippocampal but not in the prefrontal LFP. After defining DOWN- and UP-states, previously selected slow oscillation periods were post hoc checked for the occurrence of UP- or DOWN-states in at least 70\% of time. Periods that did not meet this criterion were excluded from the analysis. Cells were included in this analysis if at least one slow oscillation period of $>10 \mathrm{~s}$ was detected.
DOWN- and UP-states were segmented into 20 bins each, and firing rate histograms were calculated. Peak firing of each cell was defined as the first bin when the firing exceeded $90 \%$ of the maximum difference of firing between any bins in the UP- and DOWN-states. The time lag from transition was calculated as the time difference between the DOWN/UPstate transition and the time of peak firing. Firing increase was detected if the mean firing rate in a bin was significantly higher than the mean firing rate in at least one of the DOWN-state bins (Kruskal-Wallis ANOVA, multiple comparisons with Bonferroni adjustments, $\alpha=0.05$ ). The frequency distributions of significant firing increase were compared by Kolmogorov-Smirnov tests corrected by the Holm-Bonferroni method. Average firing rates during UP-states of slow oscillations were calculated as the total number of action potentials divided by the absolute time of 

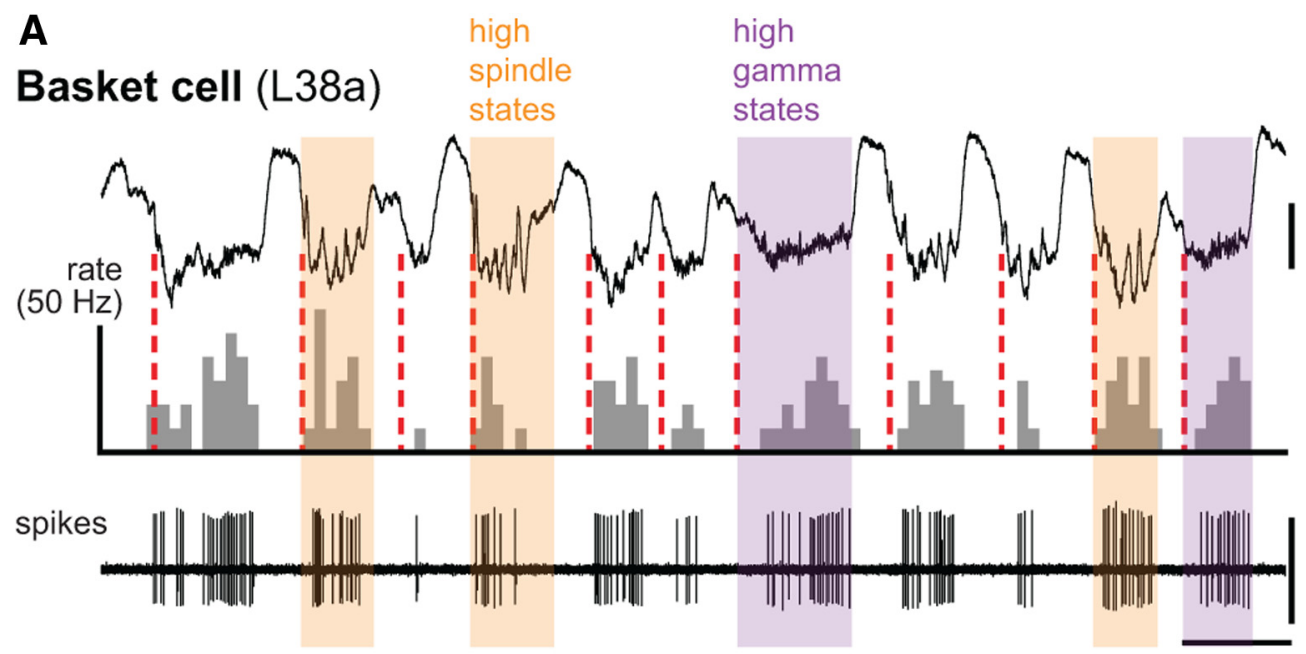

B

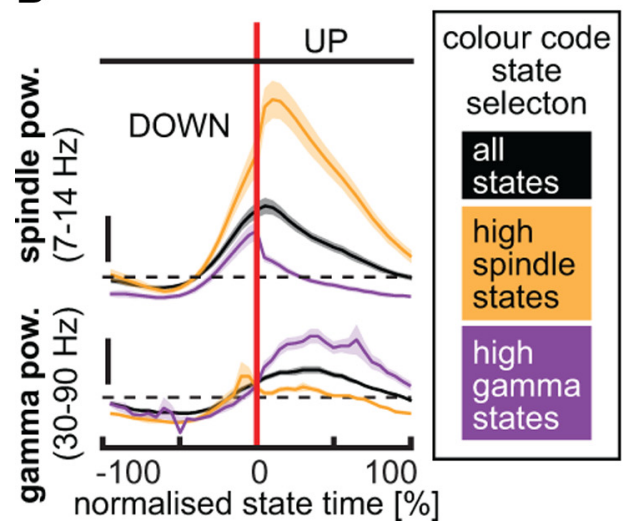

C

$$
\begin{array}{lll}
\text { axo-axonic } & \text { basket } & \text { pyramidal } \\
\text { cells } & \text { cells } & \text { cells } \\
(n=5) & (n=19) & (n=19)
\end{array}
$$

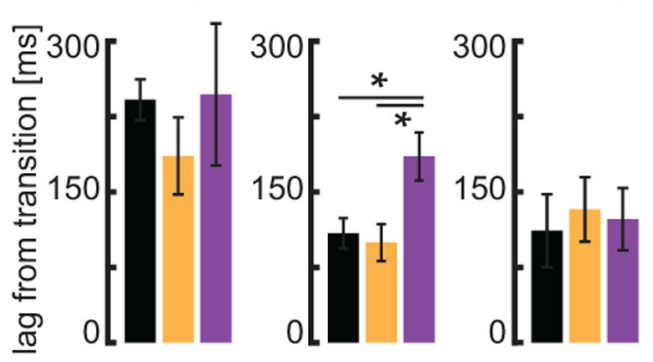

Figure 3. Firing patterns of axo-axonic, basket and pyramidal cells during gamma and spindle oscillations nested within UP-states $\boldsymbol{A}$, Recording of a basket cell during UP-states with spindle or gamma oscillations. $\boldsymbol{B}$, Mean power of spindle and gamma oscillations is shown for all UP-states (black) and preselected UP-states with high-power spindle (orange) or gamma (purple) oscillations. C, Basket cells fire significantly later during UP-states with high gamma and low spindle power. (Friedman ANOVA and Dunn's posthoc test, ${ }^{*} p<0.05$ ). All values shown as mean \pm SEM. Calibration: A, $1 \mathrm{~s}, 1 \mathrm{mV}$.

UP-states analyzed. For correlations between firing rate and spike timing, the firing rates and time lags from DOWN- to UP-state transition were calculated for each cell and for 10 different slow oscillation cycle lengths ranging from 500 to $1500 \mathrm{~ms}$. This time range included $94 \%$ of all slow oscillation cycles of all experiments analyzed. On the population level, $z$-scored firing rates and time lags were correlated using Spearman rank correlations.

UP-states were subdivided according to the profile of their main nested frequency bands of the spindle $(7-14 \mathrm{~Hz})$ and gamma $(30-90 \mathrm{~Hz})$ oscillations. Mean spindle and gamma power was evaluated by a continuous wavelet transform using a complexed Morlet (1-1.5) wavelet function, transformed to $z$-score, segmented into each 20 bins per state and averaged over all states per recording of each cell. UP-states were classified as spindle-dominated states, if the spindle power during the respective UP-state exceeded the mean spindle power plus $0.5 \mathrm{SD}$ calculated over all UP-states. The same threshold for the gamma power was used for defining gamma-dominated UP-states. Note that UP-states with high spindle power had a lower power in the gamma frequency range compared with all UP-states. Similarly, UP-states with high gamma power had a lower power in the spindle frequency band. Time lags of peak firing from transition were compared between all states, spindle- and gammadominated states by paired testing (Friedman ANOVA, Dunn post hoc test, $p<0.05)$. Recordings with $<50 \mathrm{UP}$-states and without any recorded spike during either spindle- or gamma-dominated UP-states were not included into the analysis.

All parameters, unless otherwise stated, were compared between different cell types using a Kruskal-Wallis ANOVA followed by multiple comparisons with Bonferroni adjustments with $\alpha$ set to 0.05 . Spike arti- facts were removed from the LFP by cutting out 3-5 sampling points around the detected peak of the spikes, and these points were interpolated. To avoid edge effects, all spikes being closer than $1 \mathrm{~s}$ to the start or end of each recording were excluded. Analyses were done with customwritten, MATLAB-based programs (MathWorks), except in the case of the Friedman ANOVA test, which was performed in GraphPad Prism 5.01 .

\section{Results}

Recording and identification of axo-axonic, basket, and pyramidal cells

We recorded the activity of 5 identified axo-axonic and 26 other $\mathrm{PV}$-expressing cells in the prelimbic area of the medial prefrontal cortex of anesthetized rats with extracellular glass electrodes. The recorded cells were filled with Neurobiotin by juxtacellular labeling and analyzed with immunohistochemical methods. The somata of axo-axonic cells were located in layer II and their smooth dendrites characteristically branched in layer I bending at the pia (Fig. 1A). The axonal arborization, mainly located in layer II and a lesser extend in layer III with occasional branches to V, showed characteristic axonal cartridges with typical radial rows of boutons (Szentágothai and Arbib, 1974). Immunofluorescence analysis confirmed close apposition of these axon cartridges to axon initial segments of pyramidal cells (Fig. 1C). Four out of four tested axo-axonic cells were weakly immunopositive for the calcium binding protein parvalbumin (Fig. 1B). 
The other 26 recorded and labeled PVexpressing neurons had axonal arborizations in layers II-V with considerable variability between individual cells (Kawaguchi and Shindou, 1998; Hartwich et al., 2009). The axons of some cells formed boutons surrounding cell bodies as observed by light or fluorescence microscopy and correspond to previously defined basket cells (Somogyi et al., 1983). Some of the PV-positive neurons did not appear to contribute to significant somatic innervation of other neurons. Because of the heterogeneity in axonal targets (Hartwich et al., 2009), dendritic inputs and soma position, the cells referred to as "basket cells" here are likely to include a range of neuronal types. These basket cells had positions of somata located in layers II-V at $232 \pm 33 \mu \mathrm{m}$ (as measured from the border of layers I and II; $n=19)$ and deeper $(p<0.001$, MannWhitney $U$ test) than axo-axonic cells (33 $\pm 9 \mu \mathrm{m}, n=4)$.

Pyramidal cells were recorded and labeled with glass electrodes $(n=9)$ or recorded with tetrodes $(n=20)$. Some of their parameters have been reported previously (Hartwich et al., 2009) and are not repeated here.

\section{Spike-timing during cortical UP- and DOWN-states}

During slow oscillations, the firing patterns of axo-axonic cells $(n=5)$ differed from that of basket $(n=24)$ and pyramidal $(n=26)$ cells (Fig. 2$)$. Both basket and pyramidal cells significantly increased their firing rate before the onset of the UP-state, as defined here (see Materials and Methods) and measured in the LFP (Fig. 2A,B), and reached their peak firing on average at $98 \pm 14 \mathrm{~ms}$ (basket cells) and $144 \pm 28 \mathrm{~ms}$ (pyramidal cells) after the DOWN-to-UPstate transition (Fig. $2 B-D$ ). In contrast, axo-axonic cells fired significantly later than basket and pyramidal neurons $(p<0.01$, Kruskal-Wallis ANOVA; $p<0.05$ multiple comparisons with Bonferroni adjustments), with peak firing occurring on average at $242 \pm 23 \mathrm{~ms}$ after the DOWN- to UP-state transition (Fig. 2D). Additionally, the mean firing rate of axo-axonic and pyramidal cells during UP-states of slow oscillations was significantly lower than that of basket cells ( $p<0.001$, Kruskal-Wallis ANOVA; $p<$ 0.05 , multiple comparisons with Bonferroni adjustments). To elucidate whether the difference in firing rates accounts for the temporal separation of axo-axonic and basket cells during slow oscillations, we tested for a possible relationship between the time lag of peak firing from the DOWN-to-UP-state transition and the firing frequency during slow oscillations. For axo-axonic and basket cells the time lag from transition was not correlated with the firing frequency (Spearman rank correlations, axoaxonic cells: $r=0.07, p>0.05$, basket cells: $r=-0.07, p>0.05$, pyramidal cells: $r=-0.01, p>0.05)$.

Spindle and gamma oscillations are coupled to the early and middle stages of UP-states, respectively. To test whether the occurrence of these two oscillations influences the firing of the different cell types, we selected UP-states with high-power spindle or gamma oscillations (Fig. 3A). The spike timing of axo-axonic and pyramidal cells were not different between these preselected UP-states and all UP-states ( $p>0.05$, Friedman ANOVA), but basket cells fired significantly later $(p<0.01$, Friedman ANOVA; $p<0.05$ Dunn's post hoc test) during UP-states with high gamma and low spindle power (Fig. $3 B, C$ ). These results indicate that the firing of PV-expressing basket cells is strongly coupled either to spindle oscillations occurring at the beginning of the UP-state or to gamma oscillations at the middle of the UP-state. In contrast, axo-axonic cells fire little during spindle oscillations at the beginning of the UP-state and fire always at the middle of the UP-state together with gamma oscillations, suggesting a cell-type and network oscillation-specific control of pyramidal neurons during slow oscillations.

\section{Tail pinch-induced change of firing}

In some recordings we applied a pinch to the tail of the rat during slow oscillations, which induced a switch in brain state characterized by theta oscillations in the hippocampus and a lowamplitude desynchronized field potential in the prelimbic cortex (Fig. 4A). Following each tail pinch, a significant increase of firing ( $p=0.029$, Wilcoxon signed rank test, $n=4$ pinches from 4 different rats) was observed in all tested axo-axonic cells (Fig. $4 A, B)$. Basket cells as a population $(p>0.05$, Wilcoxon test, $n=$ 23 tail pinches recorded from 19 cells), and pyramidal cells ( $p>$ $0.05, n=62$ tail pinches from 29 cells, ) did not change their firing rate (Fig. $4 B$ ). Individual basket cells showed variable responses during tail pinch. Axo-axonic and basket cells recorded were lo- 
cated in different layers and therefore the effect observed in individual cells could be due to their laminar location. However, we did not observe a significant correlation $(r=0.048, p>0.8)$ between the firing rate change and the laminar position of the somata, or the axonal patterns of basket cells. The synaptic targets of two basket cells showing different degrees of activation by pinch (L93, before/during pinch firing frequency ratio 2.2, $n=19$ synapses; L 80 , ratio $1.1, n=13$ synapses) tested by electron microscopy in layer III were similar in that $\sim 80 \%$ of postsynaptic elements were dendritic shafts and the rest dendritic spines and rarely somata. However, we cannot exclude the existence of different types of PV-expressing basket cells, which change their firing differentially during tail pinch. We found no significant difference ( $p>0.05$, Mann-Whitney $U$ test) in firing activity during tail pinch and hippocampal spontaneous theta episodes for axo-axonic, basket and pyramidal cells (Fig. 2B).

\section{Discussion}

Using in vivo extracellular recording and juxtacellular labeling, we have shown that the parvalbumin-expressing and fast-spiking interneurons, axo-axonic and basket cells, have different firing dynamics in vivo. Different dendritic arborizations and location of their somata indicate differences in their synaptic inputs. The partial overlap in their axonal fields suggests that basket cells and axo-axonic cells converge onto some of the same pyramidal cells, supporting their differential control of the spike-timing of pyramidal cells.

\section{Effect of a tail pinch on neuronal activity in the medial prefrontal cortex}

Prefrontal neurons integrate information from many cortical areas, receive glutamatergic thalamocortical innervations (Kubota et al., 2007), and noradrenergic (Kawaguchi and Shindou, 1998), dopaminergic (Gao et al., 2003) as well as GABAergic, glutamatergic and cholinergic inputs from the brainstem (Yamaguchi et al., 2011) and the basal forebrain (Manns et al., 2003). During recordings of neuronal activity in the medial prefrontal cortex, we used a tail pinch as an arousal signal to switch the brain state from slow to theta oscillations. The tail pinch caused a strong increase of firing in all tested axo-axonic cells. Notably, the recorded prefrontal pyramidal neurons, on average, did not increase their firing rate during tail pinch. This may be explained by the increased firing of axo-axonic cells, suggesting their inhibitory role as proposed previously (Somogyi, 1977; Zhu et al., 2004; Glickfeld et al., 2009; Woodruff et al., 2011). However, a pyramidal cell state-dependent excitatory role in vitro has also been suggested (Szabadics et al., 2006). Thus, inhibition by axo-axonic cells might counteract excitatory inputs from cortical and subcortical areas to allow firing only by the most activated pyramidal cells, which form neuronal assemblies with the onset of arousal.

\section{Spike-timing of axo-axonic, basket, and pyramidal cells during slow oscillations}

During slow oscillations (Cossart et al., 2003; Steriade and Timofeev, 2003; Huguenard and McCormick, 2007; Puig et al., 2008), we found that at the transition from DOWN- to UP-states, when spindle oscillations occur, pyramidal and basket cells increase their firing rate earlier than axo-axonic cells. This observation is supporting previous data showing that the firing of PV-expressing interneurons during slow oscillations can be divided into two distinct groups (Puig et al., 2008). The increase in spindle phase-locked firing of basket cells (Hartwich et al., 2009) promotes pyramidal cell synchrony without the participation of axo-axonic cells, which are mostly silent at this time. During UP-states that are dominated by gamma oscillations, the firing of basket cells shift to later phases of the UP-state, when gamma oscillations are most prominent. This indicates that basket cells may be a major generator of both spindle and gamma oscillations. The different spike-timing of basket cells during UP-states with either spindle or gamma oscillations might be a consequence of distinct excitatory inputs from thalamic or local cortical sources, respectively. However, it remains to be established how basket and axo-axonic cells fire during slow oscillations in drugfree animals.

The exclusive innervations of axon initial segments or somatodendritic domains, together with their distinct temporal dynamics, demonstrated here in vivo, support a differential role for axo-axonic and basket cells in the control of pyramidal cell activity that breaks down in schizophrenia (Lewis et al., 2005; Fazzari et al., 2010).

\section{References}

Atallah BV, Bruns W, Carandini M, Scanziani M (2012) Parvalbuminexpressing interneurons linearly transform cortical responses to visual stimuli. Neuron 73:159-170.

Buzsáki G (2006) Rhythms of the brain. New York: Oxford UP.

Cardin JA, Carlén M, Meletis K, Knoblich U, Zhang F, Deisseroth K, Tsai LH, Moore CI (2009) Driving fast-spiking cells induces gamma rhythm and controls sensory responses. Nature 459:663-667.

Cossart R, Aronov D, Yuste R (2003) Attractor dynamics of network UP states in the neocortex. Nature 423:283-288.

Csicsvari J, Hirase H, Czurko A, Buzsáki G (1998) Reliability and state dependence of pyramidal cell-interneuron synapses in the hippocampus: an ensemble approach in the behaving rat. Neuron 21:179-189.

Fazzari P, Paternain AV, Valiente M, Pla R, Luján R, Lloyd K, Lerma J, Marín O, Rico B (2010) Control of cortical GABA circuitry development by Nrg1 and ErbB4 signalling. Nature 464:1376-1380.

Gao WJ, Wang Y, Goldman-Rakic PS (2003) Dopamine modulation of perisomatic and peridendritic inhibition in prefrontal cortex. J Neurosci 23:1622-1630.

Glickfeld LL, Roberts JD, Somogyi P, Scanziani M (2009) Interneurons hyperpolarize pyramidal cells along their entire somatodendritic axis. Nat Neurosci 12:21-23.

Hartwich K, Pollak T, Klausberger T (2009) Distinct firing patterns of identified basket and dendrite-targeting interneurons in the prefrontal cortex during hippocampal theta and local spindle oscillations. J Neurosci 29: 9563-9574.

Huguenard JR, McCormick DA (2007) Thalamic synchrony and dynamic regulation of global forebrain oscillations. Trends Neurosci 30:350-356.

Kawaguchi Y, Shindou T (1998) Noradrenergic excitation and inhibition of GABAergic cell types in rat frontal cortex. J Neurosci 18:6963-6976.

Kubota Y, Hatada S, Kondo S, Karube F, Kawaguchi Y (2007) Neocortical inhibitory terminals innervate dendritic spines targeted by thalamocortical afferents. J Neurosci 27:1139-1150.

Lasztoczi B, Tukker JJ, Somogyi P, Klausberger T (2011) Terminal field and firing selectivity of cholecystokinin-expressing interneurons in the hippocampal CA3 area. J Neurosci 31:18073-18093.

Lewis DA, Hashimoto T, Volk DW (2005) Cortical inhibitory neurons and schizophrenia. Nat Rev Neurosci 6:312-324.

Lovett-Barron M, Turi GF, Kaifosh P, Lee PH, Bolze F, Sun XH, Nicoud JF, Zemelman BV, Sternson SM, Losonczy A (2012) Regulation of neuronal input transformations by tunable dendritic inhibition. Nat Neurosci 15: 423-430.

Manns ID, Alonso A, Jones BE (2003) Rhythmically discharging basal forebrain units comprise cholinergic, GABAergic, and putative glutamatergic cells. J Neurophysiol 89:1057-1066.

Pinault D (1996) A novel single-cell staining procedure performed in vivo under electrophysiological control: morpho-functional features of juxtacellularly labeled thalamic cells and other central neurons with biocytin or Neurobiotin. J Neurosci Methods 65:113-136. 
Puig MV, Ushimaru M, Kawaguchi Y (2008) Two distinct activity patterns of fast-spiking interneurons during neocortical UP states. Proc Natl Acad Sci U S A 105:8428-8433.

Royer S, Zemelman BV, Losonczy A, Kim J, Chance F, Magee JC, Buzsáki G (2012) Control of timing, rate and bursts of hippocampal place cells by dendritic and somatic inhibition. Nat Neurosci 15:769-775.

Somogyi P (1977) A specific 'axo-axonal' interneuron in the visual cortex of the rat. Brain Res 136:345-350.

Somogyi P, Kisvárday ZF, Martin KAC, Whitteridge D (1983) Synaptic connections of morphologically identified and physiologically characterized large basket cells in the striate cortex of cat. Neuroscience 10:261-294.

Steriade M, Timofeev I (2003) Neuronal plasticity in thalamocortical networks during sleep and waking oscillations. Neuron 37:563-576.

Szabadics J, Varga C, Molnár G, Oláh S, Barzó P, Tamás G (2006) Excitatory effect of GABAergic axo-axonic cells in cortical microcircuits. Science 311:233-235.
Szentágothai J, Arbib MA (1974) Conceptual models of neural organization. Neurosci Res Program Bull 12:305-510.

Tamás G, Buhl EH, Lörincz A, Somogyi P (2000) Proximally targeted GABAergic synapses and gap junctions synchronize cortical interneurons. Nat Neurosci 3:366-371.

Vida I, Bartos M, Jonas P (2006) Shunting inhibition improves robustness of gamma oscillations in hippocampal interneuron networks by homogenizing firing rates. Neuron 49:107-117.

Woodruff AR, McGarry LM, Vogels TP, Inan M, Anderson SA, Yuste R (2011) State-dependent function of neocortical chandelier cells. J Neurosci 31:17872-17886.

Yamaguchi T, Wang HL, Li X, Ng TH, Morales M (2011) Mesocorticolimbic glutamatergic pathway. J Neurosci 31:8476-8490.

Zhu Y, Stornetta RL, Zhu JJ (2004) Chandelier cells control excessive cortical excitation: characteristics of whisker-evoked synaptic responses of layer $2 / 3$ nonpyramidal and pyramidal neurons. J Neurosci 24:51015108 . 